\title{
Consciousness From a Learning Perspective
}

\author{
Onne Gorter
}

Dec 2017

\begin{abstract}
For a long time humans have wondered about consciousness. It's a problem not easily investigated with the tools of science. All the while the question is becoming increasingly more pressing with the emergence of AI systems like self-driving cars. This paper will argue that learning creates subjectivity. And that the way the brain learns requires self-observation and causes consciousness.
\end{abstract}

\section{Introduction}

There are two hard problems related to consciousness.

1. We have no device that can measure it;

2. We have no intuition about why it arises.

Yet as human beings we are certain we have it - we experience it. And this experience is easily correlated to the brain; drugs can alter the experience; deep brain stimulation can set on feelings of anger or love or pleasure[1]; brain damage can diminish or radically alter it. So why do 86 billion neurons, connected by trillions of synapses, feel like something? Do other, simpler, brains feel like something? Could a computer brain feel like something[2]? Or as Nagel[3] asks, what is it like to be a bat?

We will show that subjectivity comes from learning and that the brain learns by self-observing. Chalmers[4] asks why we are conscious, we prefer to ask that question in reverse, why would a self-observing and subjective system not be conscious?

We will use a narrow focus on this very broad subject of consciousness to see how three levels of learning show what lies beneath. Many aspects of our brains like memory, attention, emotions, language, theory of mind are important and add to the experiences. Nevertheless, we will leave them outside of this discussion to highlight what we think is at the root. Similarly, we will touch on learning algorithms and intelligence, but not go deeply into them except when relevant to the discussion. 


\section{Level 1, Learning Creates Subjectivity}

Learning is what students do in school, but in this paper we mean something more broad, something that includes machine learning algorithms[5].

At the highest level, we can describe a learning system as internal state connected to an environment through input, output and feedback. Where the system updates its state based on a negative signal in the feedback, such that future input causes different output and less negative feedback. This way, the system starts to recognize differences in input and learns how to output better responses.

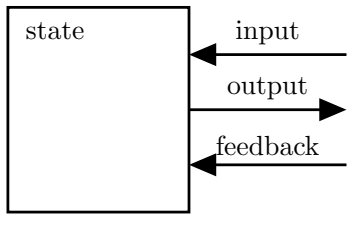

Figure 1: Direct learning

Or in more anthropomorphic terms to show this creates subjectivity: the system learns its relationship to different patterns in the environment and their meaning with respect to output and feedback.

It must be pointed out that in our description of learning, the feedback and how that generates signals, can be considered the source of subjectivity. But the signal can be very simple, while the internal state and the environment are very complex. Take evolution for example, feedback is solely based on whether an entity replicates or not.

A second note, the three signals, input, output and feedback, don't have to be very explicitly or physically present. Input can serve as feedback. Or again in the evolution example, we can talk about feedback only when we describe it as a system and see how failure to replicate is a feedback effect.

Two examples:

1. A cave is a geological oddity, caused by mindless processes like erosion. But to people who have learned that protection from the element is important, and that a solid roof and walls help, they recognize a cave can be a shelter, a home.

2. A primitive microbe culture has evolved the ability to metabolize a new group of molecules. To this strain those molecules are now a food.

Labels like "home" or "food" are subjective labels, a relationship between a system and its environment. We, with our intellect, have no problem identifying certain chemical groups as food for microbes. But the point made here is that the microbes themselves recognize this too. Not consciously and depending on 
their behavior maybe not even at an individual level, but as colonies they thrive around the food and not elsewhere.

\section{Level 2, Self-Observing}

The brain learns differently, it has internalized the input, output and feedback; it is anticipating these before they happen so it can react to situations before it's too late. Or put in another way, the brain simulates a large part of the environment and is constantly making predictions[6] and scanning those to see if they represent opportunities or dangers.

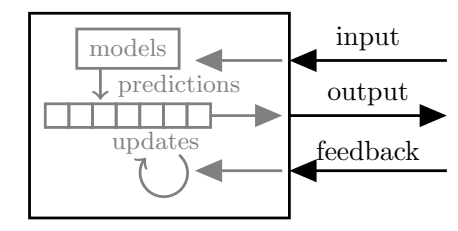

Figure 2: Predictive learning

To learn and update the models that make these predictions, and to learn the effectiveness of its choices, the brain has to keep track of these two over time. So it can compare the outside world with its predicted world; outside feedback with its predicted feedback. When there are any surprises it updates its models or its preferences, if possible. The brain is continuously observing its environment and itself.

What the brain learns is a model of the outside world; of internal signals like hunger, pain, muscle positions; and of mental states like fear, being happy, feeling save. These models are what it uses to predict. To evaluate one choice over another, it weights the potential future state and how good or bad it feels based on built-in instincts or past experiences. The various elements of these models are mental representations of the world and of internal states. All the mental models together, how they are used to predict and make choices, we can call the mind.

A few notes on this. By internalizing the signals, the feedback signal is no longer solely dictated by the environment. Instead what is negative or positive can be determined by the brain (or body) itself.

A note on mental states and representations. States like fear or happiness are often accompanied by chemicals in the brain or body. But whether or not a state has a physical carrier like that is not relevant, all neuronal activity is physical and can relay state from one moment to the next. 


\section{Level 3, Intelligence}

We cannot be sure if conscious experience requires intelligence. But we can be sure that with it the number of subjective experiences that can be had increases a lot.

A key aspect of intelligence is the ability to use the models and predictive machinery independent of any current situation. To imagine novel situations, actions and reactions. A second important aspect is to recognize features of an object as something separate from the object and the ability to compare such features abstractly and by analogy[7]. For example a surface can be rough or one can have a rough day.

The ability to imagine novel situations means the mind is able to compare the current situation to imagined, more ideal states. Without such there can be no experience of unfairness; or satisfaction from accomplishing a goal not rewarded by the brain or body.

For a mind to be self-aware it will need many representations of itself. Limited intelligence limits the minds self-image. For a mind to know itself it must be able to reflect on ideas, beliefs, long-term goals.

On a more philosophical note. A direct learning system has its goals dictated. A brain that has internalized the feedback signal, can have goals not dictated by the environment but by the brain or the body. An intelligent mind can set its own goals, and to some extent override those dictated by the environment or the brain.

\section{Are the Lights On?}

From these three levels of learning systems, we see how something physical and mechanical, like a computer, or a brain, can develop into a mind with subjective experiences and mental models; with ideas of a self, of goals, of purpose; that looks at the world and overlays meaning, recognizes potential dangers and potential rewards.

Table 1: Summarizing the three levels of learning.

\begin{tabular}{cll}
\hline level & type & yields \\
\hline 1 & direct & subjectivity \\
2 & predictive & models, choices, self-observation \\
3 & intelligent & self-awareness, goal-awareness \\
\hline
\end{tabular}

Only one question remains, are the lights on? If we were to build such a mind on a computer, something close to a human mind, could it be merely responding 
to negative feedback (pain), but not actually experiencing (feel) anything? Could it be a philosophical zombie? Could the lights still be off? Like Dennett[8] and others, we don't think that is logically possible.

Table 2: The objectively observables verses their subjective experiences.

\begin{tabular}{ll}
\hline objective & subjective \\
\hline responds to pain by trying to avoid it & recognize pain as bad \\
tries to avoid causes of pain & recognize situations as bad \\
tells us when it is in pain & aware when different mental states arise \\
tells us it wants the pain to stop & aware there are better and preferred mental states \\
tells us how painful an experience was & can recall past mental states and compare them \\
\hline
\end{tabular}

Such a mind will think it has experiences, will be able to reflect on those experiences, report on those experiences. How can such a mind be wrong about its own experiences? Especially if we see what information processing architecture lies beneath to support those experiences. If we push this mind to extreme doubt, it would be correct when it concludes: I doubt, therefore I exist.

"Cogito ergo sum"

It seems that when a system creates a flow of information in the form of constant predictions, evaluation and self-observation, it creates a mind - it creates a fundamental shift in frame of reference to the first person.

\section{Discussion}

There are a wide range of architectures imaginable that process information in such a way it all data is integrated into a first-person perspective. And until we get counter indicators, we should assume that it feels like something to be such a system.

On the other hand, brains can probably be preprogrammed so much that no learning is necessary or possible, such that data is not interpreted or related to a first-person perspective. Truly a robot interacting with the world but without subjective experiences. It is tempting to think evolution would have pre-programmed many brains that way and that the human brain is special. But research on animal consciousness shows many animals from different groups are capable of learning, like vertebrates, many arthropods (includes insects) and some mollusks[9].

In the beginning, we stated there is no objective measure of consciousness. We can also add that there will never be such a thing because consciousness is a systems effect. We can analyze a system to see if it implements a certain information processing architecture. But we can never place ourselves inside and feel its subjective experiences or lack thereof. 
We can illustrate that point with a few examples of systems. The moon orbits the earth, but an orbit is not a measurement, it is a conclusion from a systems analysis. Similarly, being alive is not a measurement but a conclusion that a living system is still functioning properly; or when too many processes have ceased and it has reached a point of no return, it has died.

Likewise, we can analyze a potentially conscious system and conclude it has the right architecture, that it behaves as we expect, that it reports to be self-aware. If it were implemented on a computer we could use a debugger to inspect and analyze the information flows. But no matter how closely we can relate the objective system states to the first person subjective experiences, we can never feel them.

There is a lot more richness to the human mind than discussed in this paper. Our emotions; our empathy; our bodies; episodic memory; all these add a lot of richness to the human subjective experience. That does pose an interesting question, what if a mind does not have all that richness, would it still be conscious? Most likely it will lead to a less emotional, less empathetic, but conscious mind. Moreover, to learn, there must be negative signals and a drive to avoid them. From this, it would seem there is a limit on how emotionless a mind can be. But the only way to know this for sure is to build such minds and study them.

\section{Conclusion}

We don't have an objective measure of consciousness. But we can recognize three levels of learning that apply to our brains and how those create an information processing system that integrates data into a first person perspective. This is how the brain is also a mind with subjective meaning and subjective experiences.

The hard problem of consciousness is that we must rely on our intuitions to judge if such a system is conscious. At the same time, it is highly likely that most systems processing information in similar ways are conscious, whether running on a brain or on a computer.

\section{References}

1. Panksepp, J. Affective consciousness: Core emotional feelings in animals and humans. 2005.

2. Searle, J. R. Minds, brains, and programs. 1980.

3. Nagel, T. What is it like to be a bat? 1974.

4. Chalmers, D. Consciousness and its place in nature. 2003. 
5. Mitchell, T. M. Machine learning; 1997; ISBN 0070428077.

6. Clark, A. Whatever next? Predictive brains, situated agents, and the future of cognitive science. 2013.

7. Hofstadter, D. R. Analogy as the core of cognition. 2001.

8. Dennett, D. The unimagined preposterousness of zombies. 1995.

9. Bronfman ZZ, G. S.; E, J. The transition to minimal consciousness through the evolution of associative learning. 2016. 